\title{
CONTROLE ON-OFF DE UM BIORREATOR UTILIZANDO O ALGORITMO DE EVOLUÇÃO DIFERENCIAL
}

\author{
G. S. SILVEIRA ${ }^{1}$, F. S. LOBATO ${ }^{2}$ \\ 1,2 Universidade Federal de Uberlândia, Faculdade de Engenharia Química \\ E-mail para contato: ${ }^{1}$ gustavo.saraiva.silveira@hotmail.com
}

\begin{abstract}
RESUMO - O uso de biorreatores na indústria química caracteriza uma linha de pesquisa de grande aplicabilidade nos dias atuais devido à alta produtividade deste tipo de processo. Para essa finalidade, determina-se a melhor forma de alimentação de substrato no biorreator de modo a maximizar a produtividade, caracterizando um problema de controle ótimo. $\mathrm{Na}$ prática, a determinação deste perfil é obtida a partir da aplicação da Teoria de Controle Ótimo. Todavia, a implementação prática desta solução é extremamente complexa, já que este perfil pode apresentar, teoricamente, grandes oscilações e singularidades. Neste contexto, a presente contribuição tem por objetivo a determinação da estratégia ótima de controle de um biorreator usando o controle on-off, isto é, o processo está ou não sendo alimentado. Os resultados obtidos com a aplicação do algoritmo de Evolução Diferencial são comparados com os obtidos a partir da Teoria de Controle Ótimo. A partir dos resultados obtidos fica evidente que mesmo sendo obtido um sub-ótimo, que esta estratégia de controle on-off configura-se como uma boa alternativa para a implementação prática dos resultados obtidos com a otimização do processo.
\end{abstract}

\section{INTRODUÇÃO}

A utilização da biotecnologia teve início com os processos fermentativos, cuja utilização remota a era Cristã. A produção de bebidas alcoólicas pela fermentação de grãos de cereais já era conhecida pelos sumérios e babilônios antes de 6000 A.C (Xavier et al., 2009). Hoje, os processos químicos e biotecnológicos são uma alternativa para atender a demanda por matérias-primas renováveis e de baixo custo, que transformam recursos fósseis e biomassa em produtos de valor agregados nas diversas áreas, tais como Petroquímica, Biotecnológica, Farmacêutica e de produção de insumos.

De um modo geral, biorreatores podem ser definidos como equipamentos no qual os substratos são transformados em produtos de interesse, através da realização de diversas reações químicas biocatalisadas por células ou enzimas. Neste contexto, a modelagem matemática do biorreator caracteriza um problema de grande interesse para a engenharia química de modo a se alcançar a máxima produtividade, através da otimização deste processo.

A resolução deste tipo de problema caracteriza o denominado Problema de Controle Ótimo (PCO), onde a partir da escolha da melhor forma de alimentação de substrato, obtêmse a maximização, por exemplo, da produtividade. A estratégia ótima consiste de arcos singulares e não singulares, onde a sequência dos instantes onde ocorrem estas transições devem ser determinados (Hong, 1986; Chen e Hwang, 1990). Neste cenário, Deb et al. (2002) propuseram uma metodologia baseada em Algoritmos Genéticos para resolver PCOs monoobjetivos com mais de uma variável de controle em biorreatores (Sarkar e Modak, 2004). 
Tradicionalmente, a resolução deste tipo de problema requer a aplicação da Teoria de Controle Ótimo para a determinação da melhor forma de alimentação de substrato. Este problema resulte em um perfil constituído por trechos singulares e não singulares. Do ponto de vista prático, a implementação desta solução não pode ser implementada, devido à complexidade deste perfil.

Diante do que foi apresentado, o objetivo deste trabalho é propor um controle on-off como alternativa ao controle singular, de modo que a sua implementação possa ser fisicamente possível. Para essa finalidade, o problema original é discretizado em termos da variável de controle, sendo o problema original transformado em um equivalente onde objetiva-se determinar o valor do vetor de variáveis de controle, bem como o instante de transição entre os mesmos. Na seção 2 é apresentada a modelagem matemática do problema de interesse. Na seção 3 é apresentada a abordagem considerada para a resolução do problema de otimização, a saber, o algoritmo de Evolução Diferencial (ED). Nas seções 4 e 5 são apresentados a metodologia e os resultados, respectivamente. Finalmente na última seção são apresentadas as conclusões deste trabalho.

\section{MODELAGEM MATEMÁTICA}

Matematicamente, o PCO relacionado com biorreatores que operam em regime batelada alimentada pode ser representado por (Lobato et al., 2007):

$$
\begin{array}{ll}
\frac{d x}{d t}=\mu x-\frac{u x}{V} & x(0)=x_{0} \\
\frac{d S}{d t}=-\sigma x+\frac{u\left(S_{F}-S\right)}{V} & S(0)=S_{0} \\
\frac{d P}{d t}=\pi x-\frac{u P}{V} & P(0)=P_{0} \\
\frac{d V}{d t}=u & V(0)=V_{0}
\end{array}
$$

em que $x, S, P$ são as concentrações de células, substrato, e produto, respectivamente, $V$ é o volume do biorreator; $u$ é a vazão de alimentação de substrato (variável de controle), $S_{F}$ é a concentração de substrato de entrada, $\mu, \sigma$ e $\pi$ representam as taxas específicas de crescimento de células, de consumo de substrato e formação de produto, respectivamente. Neste caso, a partir da determinação da melhor forma de alimentação de substrato pode-se, por exemplo, maximizar a produtividade deste processo.

\section{EVOLUÇÃO DIFERENCIAL}

Na prática, resolver um PCO consiste em resolver um problema de otimização com características bem particulares, isto é, obter o perfil da variável de controle sujeito à simulação de um sistema de equações algébrico-diferencial. Dentre os métodos para a resolução deste tipo de problema destaca-se o algoritmo de ED (Storn e Price, 1995). Esta abordagem evolutiva baseia-se na realização de operações vetoriais para a geração de candidatos à solução do problema de otimização (Kapadi e Gudi, 2004). Em linhas gerais, 
este algoritmo busca evoluir os indivíduos (vetores) da população de forma a gerar melhores candidatos para as próximas gerações. O procedimento geral do algoritmo é dado pelas seguintes etapas (Storn et al., 2005):

- inicialmente gera-se uma população inicial com $N P$ soluções factíveis (o vetor de variáveis de projeto estão dentro dos limites estabelecidos pelo usuário);

- seleciona-se três indivíduos (de forma aleatória), dentre os quais um para ser substituído (genitor principal) e os dois outros são selecionados como genitores secundários;

- a partir desta operação modifica-se o valor do indivíduo selecionado (genitor principal), a depender do valor da probabilidade de cruzamento definida pelo usuário, adicionandose ao valor atual do indivíduo a diferença entre duas outras variáveis (genitores secundários) ponderada pela taxa de perturbação $F$, também definida pelo usuário. Este procedimento representa o operador de cruzamento na ED;

- se o vetor resultante apresenta um melhor valor em termos da função objetivo, este substitui o candidato previamente escolhido, caso contrário, o candidato previamente escolhido é mantido na população.

- Este procedimento é repetido de forma que uma nova população, formada por novos e indivíduos remanescentes da população anterior, formam a nova população do algoritmo de ED. Para finalizar o algoritmo, adota-se como critério de parada o número máximo de gerações.

\section{METODOLOGIA}

A metodologia proposta neste trabalho consiste na transformação do PCO original em um problema similar através da parametrização do vetor de variáveis de controle. O intervalo de tempo $t \in\left[t_{o} t_{f}\right]$ é discretizado usando $n$ elementos de controle, $t_{i}, t_{o}=0<t_{l}<\ldots<t_{n}=t_{f}$. Em cada subintervalo de tempo, a variável de controle $u$ é aproximada por:

$$
u \equiv u_{i} \quad \text { para } t_{i} \leq t \leq t_{i+1}
$$

Com a aproximação linear por elementos proposta, o problema a ser resolvido consiste na determinação das $n$ variáveis de projeto $\left(u_{1}, u_{2}, \ldots, u_{n}\right)$. Além disso, nesta formulação também considera-se o tamanho do elemento de controle como variável, resultando em um problema com $2 n$ variáveis de projeto (o tempo final é livre). O problema proposto é resolvido usando o algoritmo de ED.

\section{RESULTADOS E DISCUSSÕES}

Para a aplicação da metodologia proposta considera-se as seguintes expressões para as $\mu, \sigma$ e $\pi$ (Ohno et al., 1976):

$$
\begin{aligned}
\mu & =0,125 S \\
\sigma & =\frac{\mu}{0,135} \\
\pi & =-384 \mu^{2}+134 \mu
\end{aligned}
$$


As condições iniciais são especificadas como: $x(0)=0,02 \mathrm{~g} / \mathrm{L}, S(0)=2,8 \mathrm{~g} / \mathrm{L}, P(0)=0 \mathrm{~g} / \mathrm{L}$, $V(0)=5 \mathrm{~L}$ e $S_{F}=2,8 \mathrm{~g} / \mathrm{L}$. Deseja-se maximizar a produtividade $\left(J_{P}\right)$, definida como a razão entre a quantidade de produto formado e a duração da operação do processo, isto é::

$$
\text { Máximo } J_{P}=\frac{P\left(t_{f}\right) V\left(t_{f}\right)}{t_{f}}
$$

No algoritmo de ED foram considerados os seguintes parâmetros (Storn e Price, 1995; Lobato, 2008): tamanho da população (50), número de gerações (250), probabilidade de cruzamento $(0,8)$, taxa de perturbação $(0,8)$ e estratégia $(E D / r a n d / 1 /$ bin $)$. Para esta aplicação são considerados três elementos de controle $(n=3)$, isto é; $t_{s 1}, t_{s 2} \mathrm{e} t$, bem como três variáveis de controle discretizadas $\left(u_{1}, u_{2}\right.$ e $\left.u_{3}\right)$, definidas para $0 \leq u \leq 2 \mathrm{~L} / \mathrm{h}$ e $V_{\max }=12 \mathrm{~L}$. Para fins de comparação do controle on-off obtido, será considerado o controle singular proposto por Sarkar e Modak (2004). Este é composto por três fases: $i$ ) $u=0$ (nenhum substrato é alimentado de modo que a concentração de células possa aumentar o mais rápido possível); $i i)$ $u=u_{\text {singular }}$ (obtido pela aplicação da Teoria de Controle Ótimo); e iii) $u=0$ (nenhum substrato é alimentado, já que o volume máximo do biorretor foi alcançado). Neste caso, esses autores determinaram os instantes em que cada uma destas fases acontece.

A Tabela 1 apresenta os resultados médios obtidos com a execução do algoritmo de ED dez vezes. Nesta tabela é possível observar que o resultado obtido pelo controle on-off é bem próximo ao obtido considerando a estratégia singular proposta por Sarkar e Modak (2004), caracterizando desta forma um sub-ótimo. Cabe ressaltar que em termos do tempo total de operação, a abordagem proposta foi capaz de reduzir esse valor em relação à abordagem singular.

Tabela 1 - Resultados obtidos com a aplicação do algoritmo de ED.

\begin{tabular}{|c|c|c|c|c|c|}
\hline \multicolumn{2}{|c|}{ Estratégia Singular } & \multicolumn{4}{c|}{ Estratégia on-off } \\
\hline$t_{f}(\mathrm{~h})$ & $J_{p}(\mathrm{~g} / \mathrm{h})$ & $t_{s 1}(\mathrm{~h})$ & $t_{s 2}(\mathrm{~h})$ & $t_{f}(\mathrm{~h})$ & $J_{p}(\mathrm{~g} / \mathrm{h})$ \\
\hline 25,9684 & 23,1258 & 8,6596 & 16,1529 & 23,8007 & 22,9275 \\
\hline
\end{tabular}

A Figura 1 apresenta os perfis (controle singular e controle on-off) de concentração de células, substrato, produto e volume, respectivamente. Conforme observado nesta figura, percebe-se que na primeira fase $(u=0)$, a concentração de células tende a aumentar e concentração de substrato tende a diminuir o mais rápido possível, o que favorece a formação de produto. Na segunda fase $\left(u=u_{\max }\right)$, substrato é alimentado no biorreator, o que favorece a redução da concentração de células e o aumento da concentração de substrato (efeito da diluição). Esta forma de alimentação continua até que o biorreator alcance o seu volume máximo, a partir do qual tem-se $(u=0)$.

Já na Figura 2 são apresentados aqui os perfis referentes a variável de controle e a produtividade considerando ambas as estratégias. 
Figura 1 - Perfis de concentração de células, substrato, produto e volume.
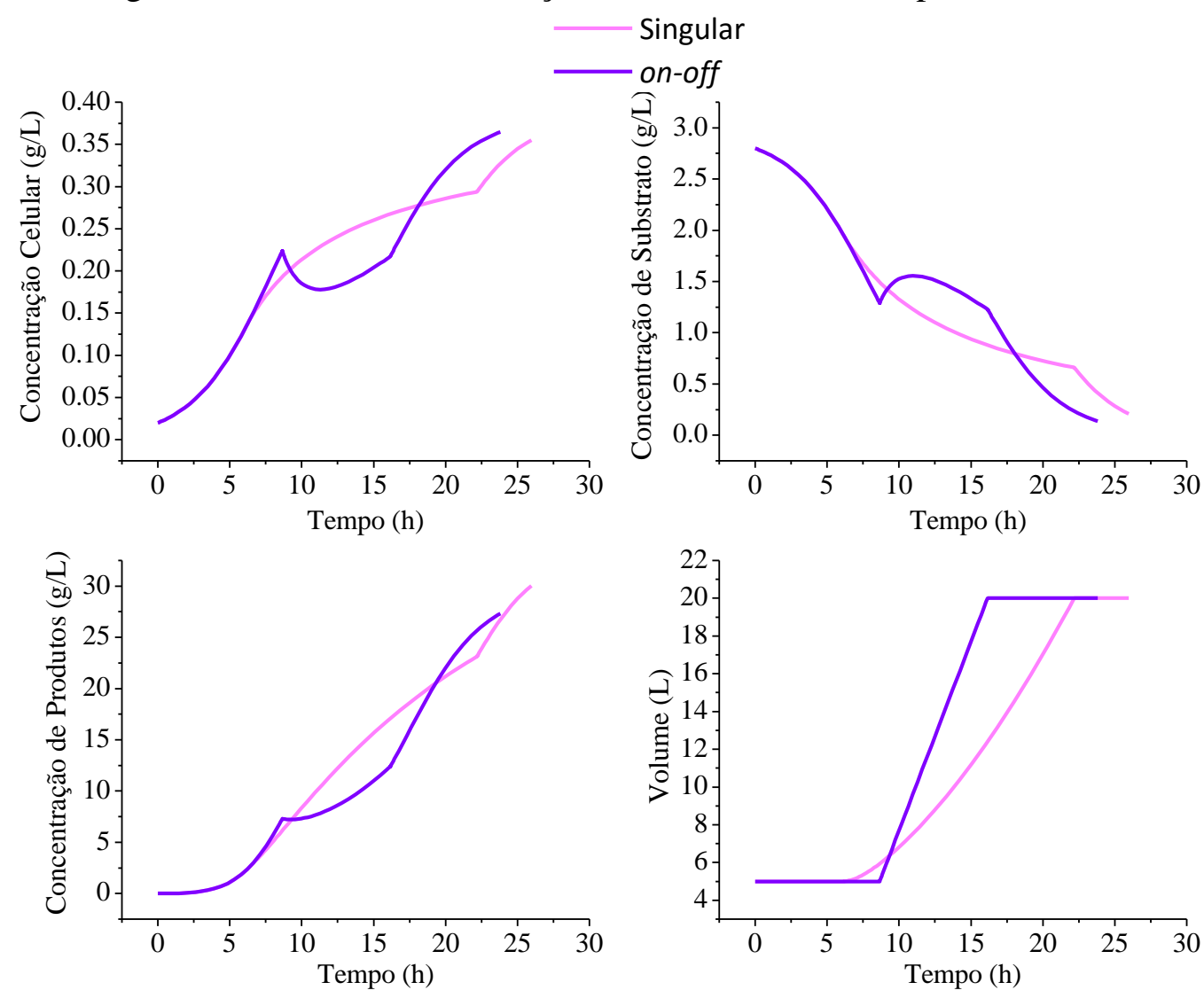

Figura 2 - Perfil da variável de controle e de produtividade.
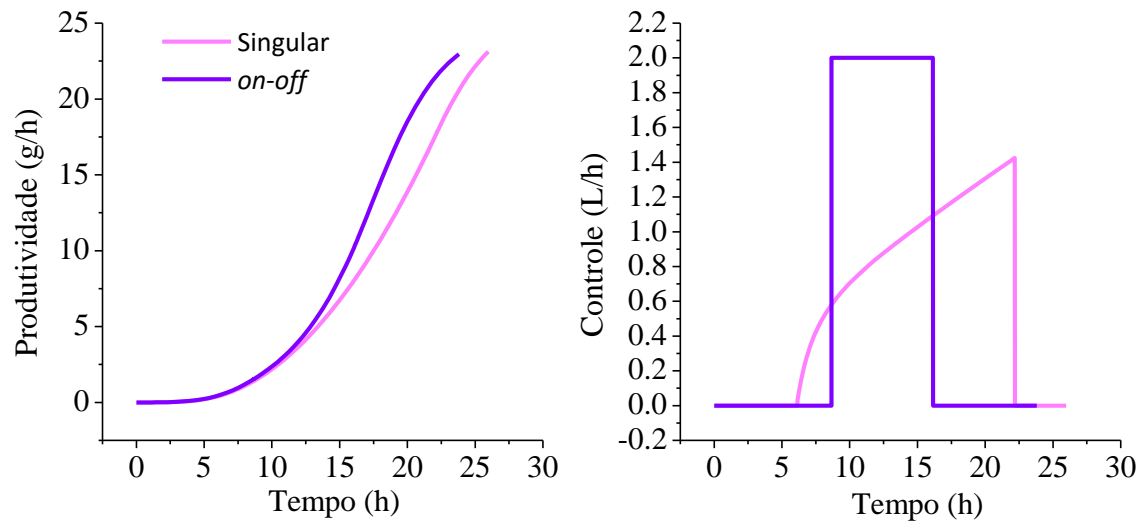

Em termos de produtividade, observa-se que ambas as estratégias têm comportamentos bem semelhantes, apesar de estratégias de controle diferentes. Na prática, o controle singular, como esperado, tem o melhor valor de produtividade, todavia, com um maior tempo de operação em relação ao controle on-off. Neste caso, observa-se uma redução de aproximadamente $7,68 \%$ no tempo total de operação do controle on-off em relação ao controle singular. Assim, apesar de uma menor produtividade, a estratégia proposta é considerada uma alternativa interessante, já que o tempo total de operação foi reduzido, sem prejudicar consideravelmente a produtividade do processo. 


\section{CONCLUSÃO}

O presente trabalho teve por objetivo o controle on-off de um biorreator considerando o algoritmo de ED. Os resultados obtidos com a metodologia proposta foram comparados com o controle singular proposto por Sarkar e Modak (2004). De forma geral, os resultados obtidos são coerentes com os da literatura. $\mathrm{O}$ valor da produtividade obtido pelo controle onoff, como esperado, foi inferior ao controle singular. Todavia, o tempo total de operação requerido pelo controle on-off é bem inferior ao requerido pelo controle singular, o que configura, em termos práticos, uma boa justificativa para a aplicação da metodologia proposta.

\section{REFERÊNCIAS}

CHEN, C.; HWANG, C., 1990, "Optimal on-off control for fed-batch fermentation processes”, Ind. Eng. Chem. Res., v. 29, p. 1869-1875.

DEB, K., PRATAP, A., AGARWAL, S., MEYARIVAN, T., 2002. A fast and elitist multiobjective genetic algorithm: NSGA-II. IEEE Transactions on Evolutionary Computation $6(2), 182-197$.

XAVIER, E. G., LOPES, D. C. N., PETERS, M. D. P., 2009. "Organismos geneticamente modificados", Archivos de zootecnia vol. 58(R), p. 15-32.

HONG , J., (1986), "General characteristics of optimal feed rate profiles for various fed-batch fermentation process", Biotech. and Bioeng., v. 38, p.1421-1431.

KAPADI, M., GUDI, R. D. Optimal control of fed-batch fermentation involving multiple feeds using Differential Evolution. Process Biochemistry, v.39, p. 1709-1721, 2004.

LOBATO, F. S. Otimização Multi-objetivo para o Projeto de Sistemas de Engenharia. Tese de Doutorado, Faculdade de Engenharia Mecânica, Universidade Federal de Uberlândia, Uberlândia-Brasil, 2008.

LOBATO, F. S., MURATA, V. V., OLIVEIRA-LOPES, L. C., STEFFEN, V. Solution of multi-objective optimal control problems with fluctuation index using differential evolution, UNESP, 2007.

OHNO, H., NAKANISHI, E., TAKAMATSU, T., 1976. Optimal control of a semi-batch fermentation. Biotechnology and Bioengineering 18, 847-864.

SARKAR, D., MODAK, J.M., 2004. Pareto-optimal solutions for multi-objective optimization on fed-batch bioreactors using non-dominated sorting genetic algorithm. Chemical Engineering Science 60, 481-492.

STORN, R., PRICE, K. Differential Evolution: A Simple and Efficient Adaptive Scheme for Global Optimization over Continuous Spaces. International Computer Science Institute, vol. 12, pp. 1-16, 1995.

STORN, R., PRICE, K., LAMPINEN, J. A. Differential Evolution - A Practical Approach to Global Optimization. Springer - Natural Computing Series, 2005. 